\title{
Measurement and Control System Design of Precision Retentivity of Rolling Linear Guide Pair
}

\author{
Jun Han, Hutian Feng, Yi Ou, Yi Liang, Aihua Yin, and Li Zu
}

\begin{abstract}
Researched measurement and control system of precision retentivity of rolling linear guide pair for the problem of precision retentivity of rolling linear guide pair. Introduced the test bed testing system respectively from the hardware structure and software design in detail, and analyzed the data of test results. The design of test system provides a platform for the on-line measurement of precision retentivity of rolling linear guide pair, provides theoretical basis, testing methods and data support for new product research and development or product improvement. It has a very important significance to improve the performance of rolling linear guide pair made in China.
\end{abstract}

Index Terms-Rolling linear guide pair, control system, hardware structure, software design.

\section{INTRODUCTION}

In recent years, with the development of $\mathrm{CNC}$ machine to high speed, high precision and long life direction, higher requirements have been put forward on the performance of rolling linear guide. As the load-bearing parts of machine tool and mechanical device, rolling linear guide pair can ensure the operation precision of machine tool and mechanical device and reduce the drive power consumption at the same time, because of its advantages of high positioning precision, small static and dynamic friction coefficient, high precision retentivity [1], it has become a key functional component of high-speed precision $\mathrm{CNC}$ equipment [2]. However, more than $80 \%$ of rolling linear guide pairs with high precision retentivity depends on import, which influences China's independent research, development and localization of high-end CNC equipment seriously [2]. In view of this situation, it is very necessary to make research on detection theory and method of precision retentivity of rolling linear guide pair, develops a test and detection device and design test and detection method, which can test rolling linear guide pair simulating actual loading conditions and can test its precision retentivity index on-line, which provides theoretical basis, testing methods and data support for new product research and development or product improvement. It has a very important significance to improve the performance of rolling linear guide pair made in China.

Manuscript received December 2, 2014; revised May 23, 2015. This work was supported by the project named China national major science and technology projects: "Common technology research and development of rolling functional components of highend CNC machine tools," (2012ZX04002021), and the National Natural Science Foundation of China (51105206).

The authors are with Nanjing University of Science and Technology, Nang Jing, Jiangsu Province, China (e-mail: hanjun7045@163.com, fenght@mail.njust.edu.cn).

\section{PRINCIPLE AND METHOD OF PRECISION RETENTIVITY MEASUREMENT}

\section{A. Principle of Precision Detection}

Precision retentivity is an important index to evaluate the mechanical properties of rolling linear guide pair, precision will directly influence the service life of rolling linear guide rail pair, thereby affecting the processing or running accuracy of machine tools and other equipments. Precision retentivity of rolling linear guide pair mainly depends on the wear resistance, pre load and dimensional stability [3]. Precision retentivity of rolling linear guide pair refers to the ability of rolling linear guide pair to retain the precision in a given precision level under specified conditions and within the stipulated time. For rolling linear guide pair, effective precision keeping time is used to describe the intensity of precision retentivity, it refers to the time that precision of rolling linear guide pair retained in the specified grades range without loss under specified conditions. For rolling linear guide pair, its precision indicators include: when slider moves, the parallelism of its top surface related to the bottom datum of the rail; when slider moves, the parallelism of its lateral surface related to the lateral datum of the rail, and the amount of change of the pre strain force in motion process [4]

The basic detection principle of precision of rolling linear guide pair index is shown in Fig. 1, Fig. 1(a) tests parallelism of rolling linear guide pair, tested guide rail pair will be fixed on special platform before testing; put the special stent on the special platform, makes the bottom datum plane of the special stent the same to the bottom datum plane of the tested guide rail pair, it's lateral side abuts on the lateral side of the tested guide rail pair; the indicator is fixed installed on the special stent, measurement head of indicator touches the center position of the tested slider's top surface and the center position of the tested slider's lateral side which is ipsilateral to the special stent's lateral installation datum plane. When testing, move the tested slider and the special stent at the same time, test the depth of parallelism of slider moving to the guide rail's datum plane on the full-length of the tested guide pair, including the depth of parallelism of the slider to guide rail's base surface A and to guide rail's lateral side datum plane $\mathrm{B}$, measures with the maximum difference data read from the indicator. Fig. 1(b) tests dynamic changes of preload drag force of rolling linear guide pair, tested guide pair are fixed when testing, use dynamometer to pull the slider horizontally and in uniform speed along the length direction of the guide rail, compare the drag force measured with the factory setting preload drag force, determine the amplitude of variation and record in percentage form [5]. 
The tested slider The indicator The special stent

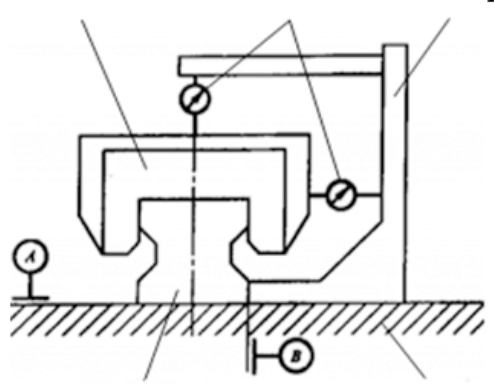

The tested guide rail

The special platform

(a)

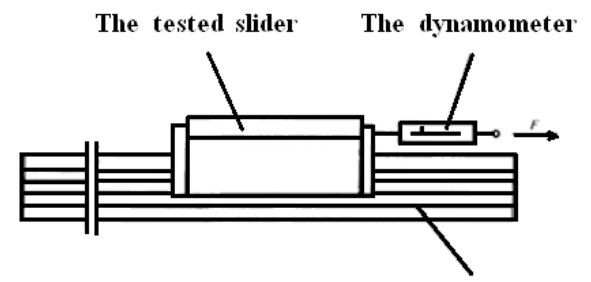

The tested guide rail

(b)

Fig. 1. Basic detection principle of precision of rolling linear guide pair.

\section{B. Measurement Method of Precision Retentivity}

The concrete implementation measurement method of precision retentivity of rolling linear guide pair, is shown as Fig. 2. For the parallelism of slider moving related to the datum of the guide rail, two groups of laser displacement sensors 1 fixed on the sensor mounting plate 2 are used to measure, the number of the laser displacement sensors in each group is two, the laser measuring head shines respectively on the side surface of the guide rail which is ipsilateral to the sensor mounting plate 2 and the surface of the guide rail keysets, the plane and the installation bottom datum are processed by the same procedure to ensure that the two plane have the same size, surface roughness and shape position tolerance. Mobile gantry crane drives two groups of laser displacement sensors 1 moving with the tested guide rail pair when moving and we can do on-line dynamic measurement of the parallelism of slider moving related to the datum of the guide rail.

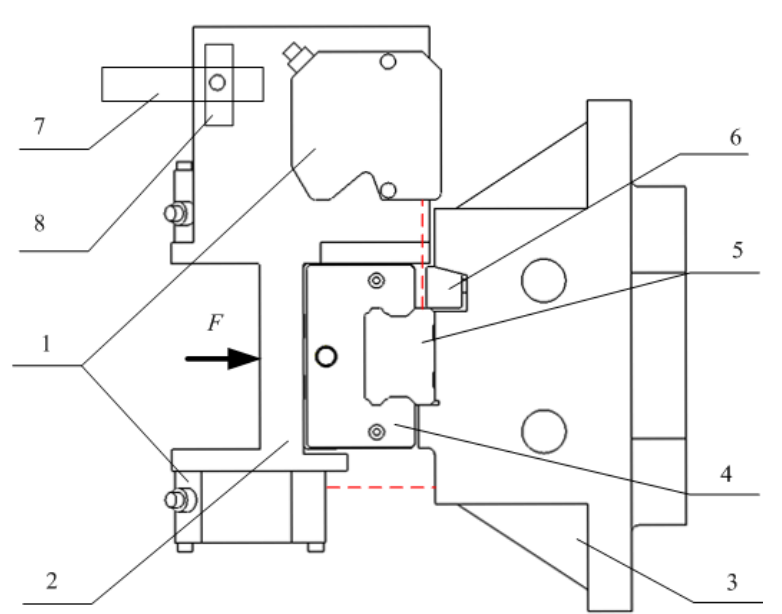

1-displacement sensor 2-sensor mounting plate 3-the guide rail keysets 4-the tested slider 5-the tested guide rail 6-briquetting 7-the sensor bracket 8-pull pressure sensor.

Fig. 2. Implementation measurement method of precision retentivity.
For the amount of change of the pre strain force in motion process of rolling linear guide pair, force sensor 8 is used to detest, one end of the force sensor 8 is fixed on the sensor mounting plate 2 and the other end is fixed on the mobile gantry crane by the sensor bracket 7 . Unloading the test load, mobile gantry crane doing uniform motion, pulling (or pushing) the slider through the sensor bracket 7 , pull pressure sensor 8 and sensor mounting plate 2 and we can measure the pre strain force [5], [6].

\section{MEChanicAl StRUCTURE OF PRECISION RETENTIVITY MEASUREMENT SYSTEM}

Test bed of precision retentivity of rolling linear guide pair is mainly comprises four parts: support structure, mobile gantry crane, loading mechanism and the drive mechanism. As shown in the Fig. 3, two tested guide pair are installed on the adapter plates on both sides, the adapter plate can be replaced according to different type of guide, use symmetric loading mechanism simulating loading the actual working condition of the tested guide rail pair from lateral. Slider table configure 4 displacement sensors and 2 vibration sensors, which test the changes of guide pair in two directions and the vibration signal when precision loss to extract the feature of precision loss.

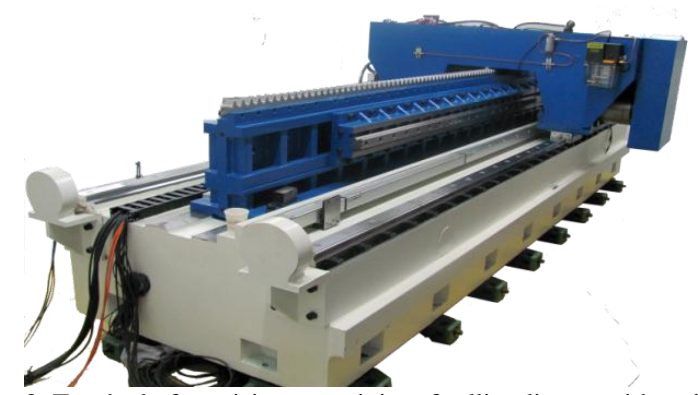

Fig. 3. Test bed of precision retentivity of rolling linear guide pair.

Designed the bed, the mobile gantry crane and the transmission mechanism by test bed, use lateral symmetrical load innovatively, this loading method can make the loading force cancel each other out, which can avoid the load acting directly on the reference guide rail pair and the bed and can improve the service life of the reference guide rail pair and the bed. Test bed can test and detect on two groups of tested guide rail pair simultaneously, this design can greatly improve the testing efficiency because the precision retentivity test time is long.

\section{MEASUREMENT SySTEM's HARDWARE STRUCTURE OF PRECISION RETENTIVITY}

\section{A. Basic Components of the Measurement and Control System}

Measurement and control system of precision retentivity of rolling linear guide pair test bed is composed of hardware system and software system, the hardware system can be divided into loading control system, motion control system and data measurement system. The software system can be divided into CNC system software and IPC software, the 
overall block diagram of measurement and control system is shown in Fig. 4.

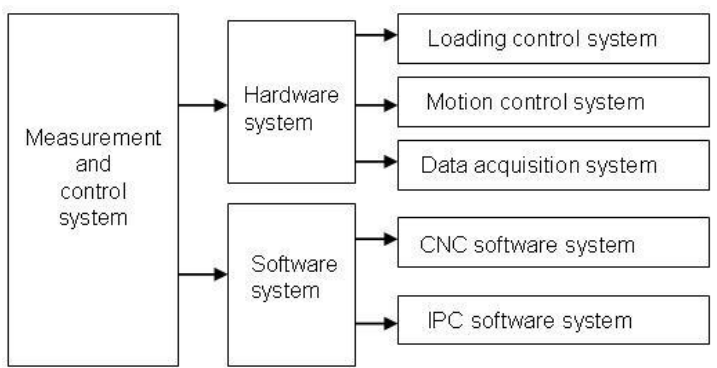

Fig. 4. The overall block diagram of measurement and control system.

\section{B. Loading Control System}

Loading control system. Choose Large power Yaskawa AC servo motor as load source because the load of the test bed is big and the require of precision is high. Use Advantech IPC(Industrial control computer) and Googol motion card as loading control system, and test and feedback the test load through two sets of high precision force sensors. When test loading, IPC sends the control signal to the servo drive by Googol motion control card, control loading servo motor starts to rotate load, when the load reaches the set value, force sensor feedback signal to the IPC, the control loading servo motor stops rotating, and keep the loading force by the self lock principle of trapezoidal screw and trapezoidal nut, loading control relationship diagram as shown in Fig. 5.

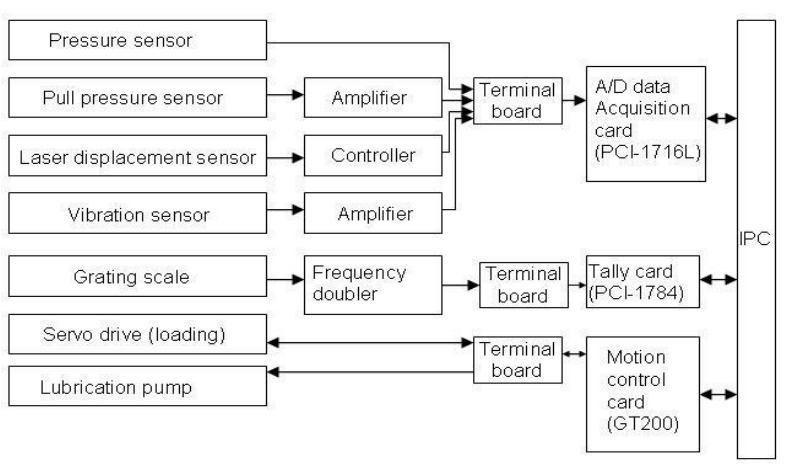

Fig. 5. Load and data acquisition hardware block diagram.

\section{Motion Control System}

Because the test bed needs to run at a high speed the in a long-term stably and should be able to control position of the tested guide rail pair accurately, the motion control system adopts Siemens CNC system. Test bed does reciprocating motion in different equivalent speed at different stages, the $\mathrm{CNC}$ system can control the stroke, speed, acceleration of gantry crane and can make the gantry crane do reciprocating motion according to the test requirements. When something emergency happens on the test bed, such as the gantry crane runs beyond the expected travel, it will firstly encounter two soft limit switches is arranged inside the CNC system and stop running; If the software limit switches lose efficacy at the same time, the gantry crane will continues to move forward and meet the hardware limit switch on both sides of the bed, signals can also be send to the CNC system and make the servo motor stop rotating, as shown in Fig. 6.

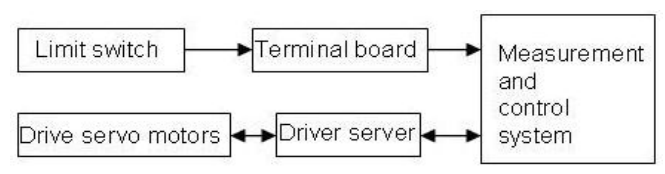

Fig. 6. Block diagram of motion control system's hardware.

\section{Data Acquisition System}

Data acquisition system of the test bed is composed of industrial control computer, A/D data acquisition card, card counting, displacement sensor, force sensor, vibration sensor and long grating etc. Test bed selects high precision laser displacement sensor to measure the depth of parallelism between the top surface of the slider and the lateral side relative to the datum plane parallelism; Select high precision force sensor and fixedly install it on the slider to move with it and dynamic pre strain can be measured online; The vibration sensor is used to detect the vibration signal of precision loss of rolling linear guide rail pair and extract the feature of precision loss; the grating reading head is fixedly installed on the tested guide rail pair and monitors and feedbacks the position of the tested guide rail pair in real-time, as is shown in Fig. 5.

\section{MEASUREMENT AND CONTROL SYSTEM SOFTWARE DESIGN OF PRECISION RETENTIVITY}

\section{A. Main Interface of Measurement and Control System Software}

The main interface of test system software is shown in Fig. 7. The main interface is divided into two parts, the menu bar and toolbar menu bar, including parameter setting, dynamic measurement, data analysis, data files, the rail profile, print output, comprehensive query, grating testing, interface testing, motor test and system maintenance etc. The toolbar is divided into parameters, loading force, parallelism, long grating, PCI-1716 board test and PCI 1784 board test. Simple icons are set up in order for easy operation.

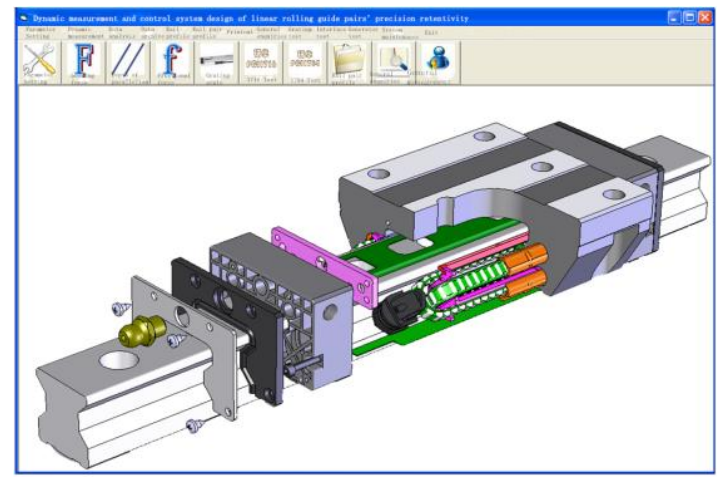

Fig. 7. The main interface of measurement and control system software.

\section{B. Parallelism Measurement}

Control system uses eight high precision laser displacement sensors, which are divided into four groups, respectively measuring parallel misalignment of two guide rail pair in vertical and horizontal plane. The corresponding interface of software measurement is shown in Fig. 8. The parallel deviation of guide rail pair is measured by displacement of 
sliding rail. In the left side of the interface is the change of displacement that relatives to the guide rail pair installation reference, in the right side of it is the specific numerical change of displacement.

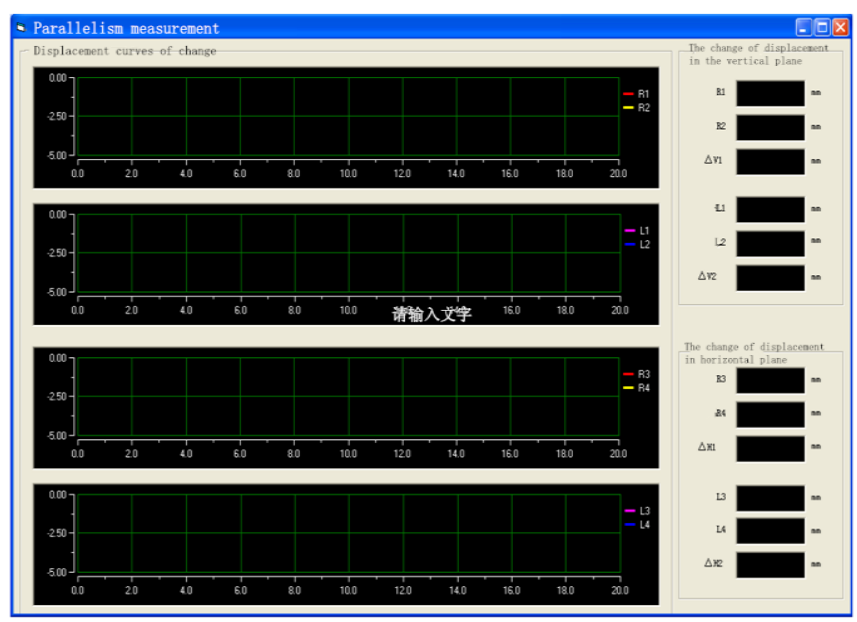

Fig. 8. Measurement interface of the parallel deviation.

\section{Detection and Control of Loading Force}

Loading system of the test bed is designed for detection and control by configuring two high precision sensors, the corresponding software program interface is shown in Fig. 9.

Firstly, input setting of loading capacity value, then we can use the control button of loading, unloading or stop operation. The interface of measurement can display the specific values of two force sensor and draw the loading force curve.

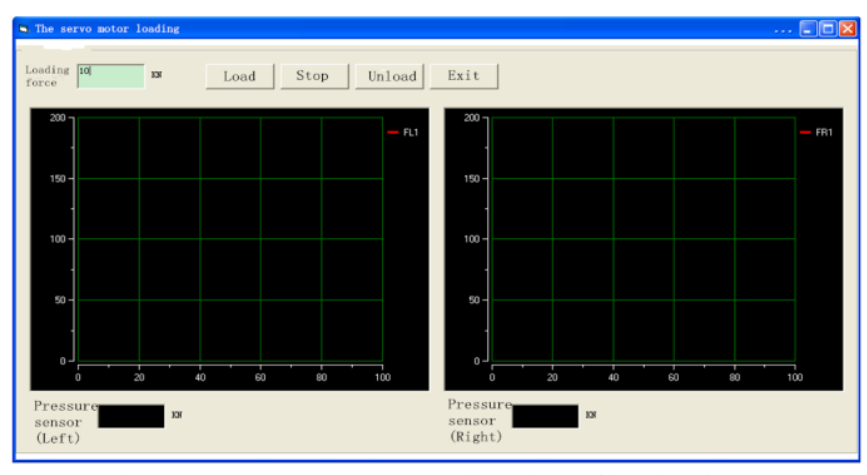

Fig. 9. Measurement and control interface of loading force.

\section{Numerical Control System Software}

The NC program of test system uses G code. It realizes the motion control of test platform by setting related parameters that controls the rotate of AC servo.

\section{ANALYSIS OF TEST RESUltS}

Combined with the designed test bed of precision retentivity of rolling linear guide pair, we made the test steps of precision retentivity of rolling linear guide pair, specific test steps are shown as Fig. 10.

The interface of parallelism measuring shows eight measured value of laser displacement sensor at the same time and updated the maximum measured value and the minimum measured value in real time in one stroke. The real-time displays about the parallelism of two groups of guide rail pair.

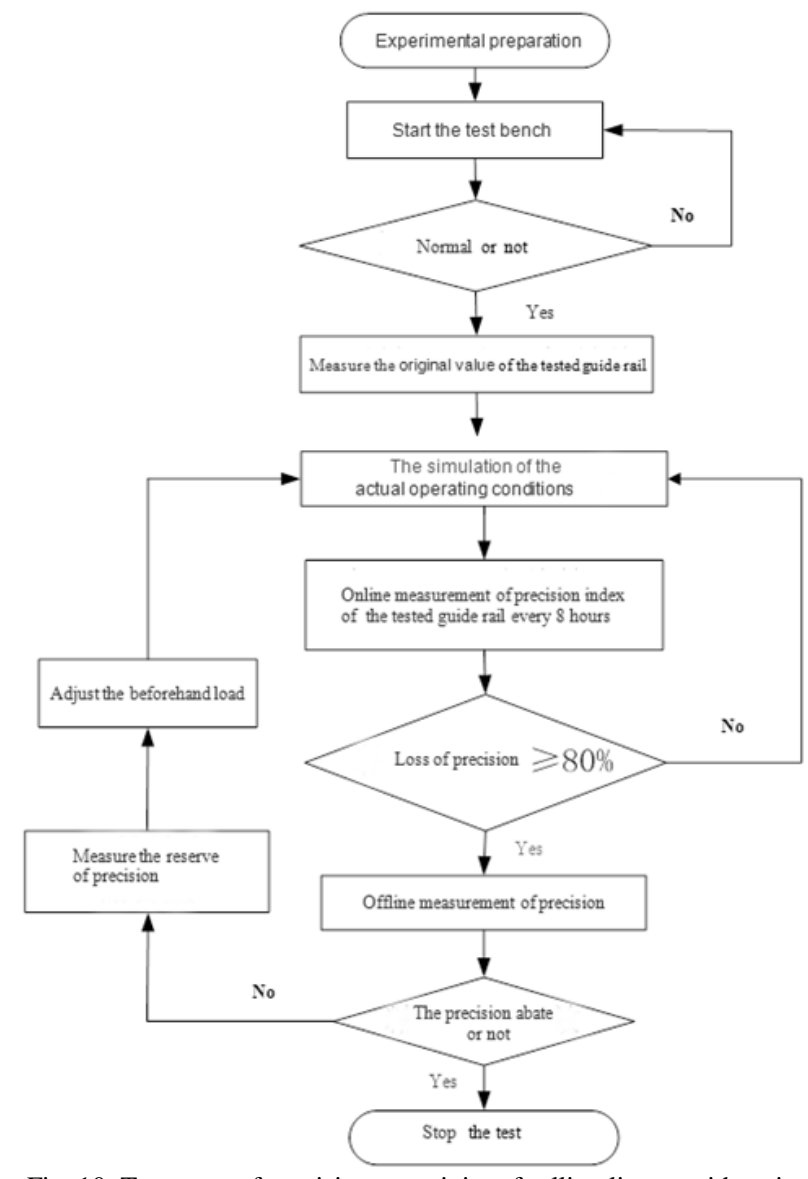

Fig. 10. Test steps of precision retentivity of rolling linear guide pair.

when the top of slider facing the bottom of the guide rails reference, the parallel degree between the side of a slide block on the ipsilateral side of rail base, the deflection angle, pitching angle relative to the initial state variation and the maximum value of yaw and pitch angle variation in one stroke by calculating.

The test bed of precision retentivity of rolling linear guide pair test will save the collected data and the analyzed data in two different database so that we can find the data and analyze them by different searching method. When we search the original data collected by sensors, we can choose the data table in integrated query, then search according to the searching request. If the search condition satisfies the uniqueness of guide, which determines the date of inspection, type and position of the guide rail. The information of the guide rail and the test information will also be shown.

Some part or all searching result will be carried out in the print statements interface. When the search is according to the measured results of one time, we can search it by date and its location. The test information and the detected information of the guide rail will be shown at the same time. This print statement is one test report. If we will search a given rail, we can use the guide number (the guide number is unique). Here will shows all of the test results and the guide information. This print statement comprises all results for precision retentivity of rolling linear guide pair test, including the parallelism of the side, parallel to the top face, the variation of yaw angle, the variation of pitch angle, equivalent load and the change of equivalent velocity with distance during the whole experiment. Precision of rolling linear guide pair testing report is shown as Fig. 11. 


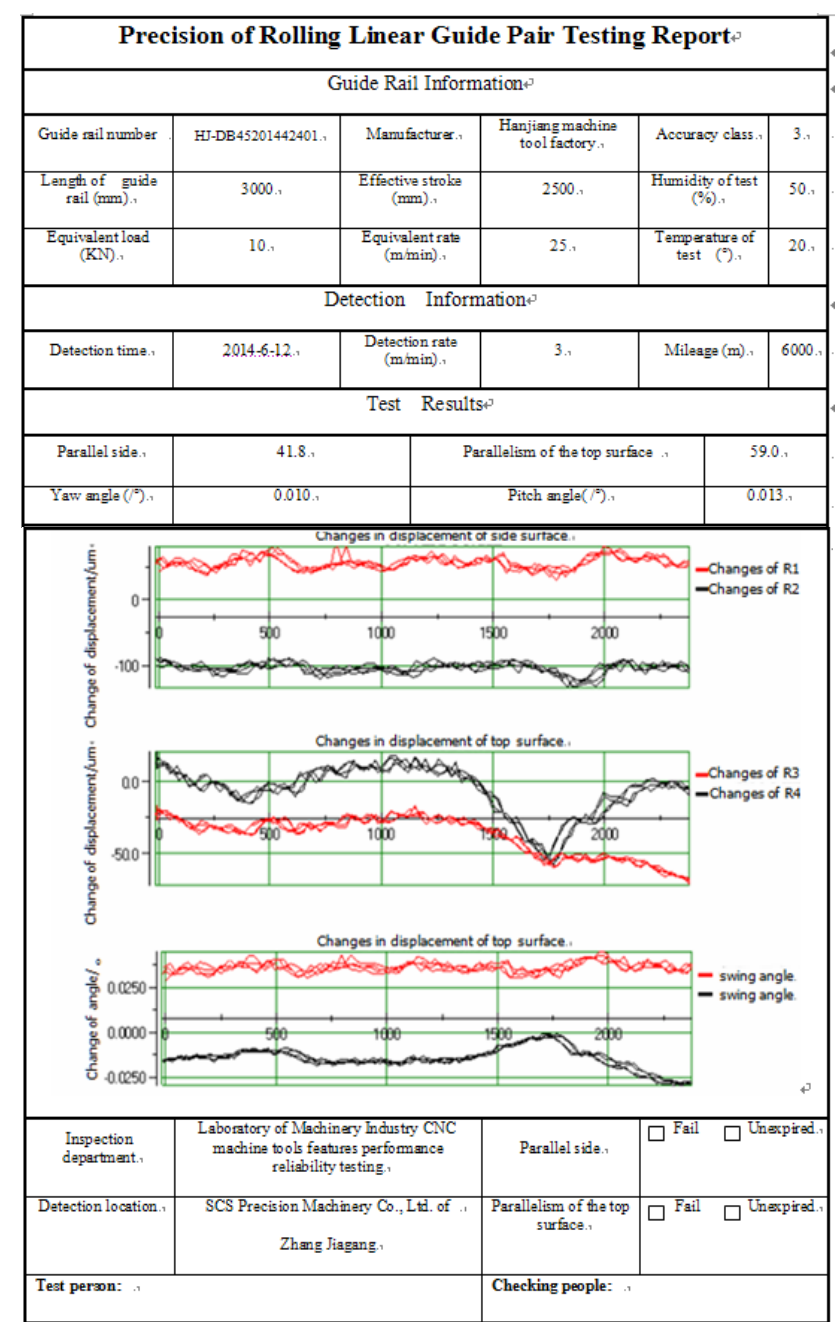

Fig. 11. Precision of rolling linear guide pair testing report.

\section{CONCLUSION}

Researched the detection method and system of implementation of precision retentivity for the problem of precision retentivity of rolling linear guide pair. Made the hardware structure and software design of measurement and control system of precision retentivity of rolling linear guide pair. Provide truly effective reference data for measuring precision retentivity of rolling linear guide pair by tests. Provide detection methods and data support for improving precision retentivity of rolling linear guide pair.

\section{REFERENCES}

[1] Z. S. Tao, X. H. Yin, X. F. Cai, and E. Zhang, "A rolling linear guide comprehensive test instrument," Modern Measurement and Test, vol. 3, pp.16-19, 1995.

[2] W. Li, "One automatic measuring method of rolling linear guide way," Modular Machine Tool \& Automatic Manufacturing Technique, vol. 3, pp. 34-37, 2010.

[3] J. B. Liu, "Micro nano processing technology in the precision stage," Chinese Academy of Sciences Institute of Electrical Engineering, 2004.

[4] JB/T, Rolling Liner Guide Pair, Part Four: Acceptance Conditions of Technology, 2006, vol. 4, pp. 71-75.

[5] Y. Zhong, "Rolling linear guide way precision to maintain basic design theory research and test platform," Master dissertation, Nanjing University of Science and Technology, Jiang Su, China, 2014.

[6] T. X. Huang, Z. Yan, and T. Zeng, "Several measurement methods of measuring straightness error of rolling linear guide of machine tool," Mechanical Engineering and Automation, vol. 2, pp. 186-188, 2008.

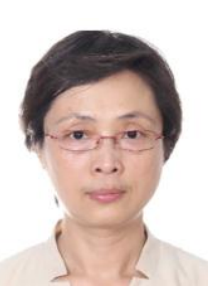

Jun Han was born in Hebei, China, on August 5 , 1963. Jun Han earned her bachelor's and master's degrees of mechanical engineering in Nanjing University of Science and Technology, Jiangsu province, China, in 1985 and 1994, respectively.

She has been teaching and researching in the field of mechanical engineering since 1985 in Nanjing University of Science and Technology. The courses she teaches are introduction to control engineering and fundamentals of robotics, fundamentals of machine and electricity transmission and control for the undergraduates. As an associate professor, she focuses on the research of precision detection and control, and robot control technology. Two of her published articles are listed here: 1). "Development of the experiment system of kinematics of 6R robot," Laboratory Research and Exploration, vol. 22, pp. 103-104, Jun. 2003, 2). "Model study of precision loss of linear ball guides," Modular Machine Tool \& Automatic Manufacturing Technique, vol. 12, pp. 33-36, Jun. 2013. One of her published book is: Comprehensive Performance Measurement Methods and Technology for Ball Screw Pair, China Machine Press, 2011. Her major field of study is precision detection and control and robot control Ms. Han joined the membership in China Electro Technical Society.

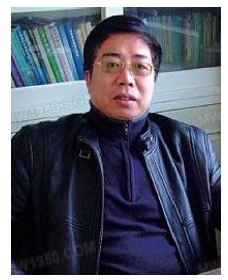

Hutian Feng was born in Jinzhou, China on January 21, 1965. In July 1986, Feng received the bachelor's degree in Zhongbei University, China in mechanical engineering. In March 1991, he received his master's degree in Nanjing University of Science and Technology, China in mechanical engineering. In September 2000, he received the doctor's degree in Nanjing University of Science and Technology, China in mechanics.

His research concerned multi-functional moving robot and mechatronic measurement and control technology. He now is in charge of the national key science and technology project: Research of Rolling Components in High-End CNC Machine Tool. He is also the vice-dean of the Mechanical and Electronic Engineering Department.

Dr. Feng is a member of Chinese Mechanical Engineering Society.

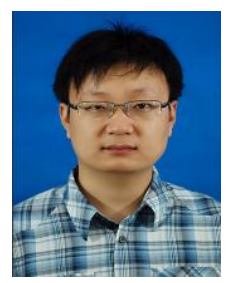

Yi Ou was born in Shaanxi, P.R. China, on August 28 1982. Ou earned his bachelor's and master's degrees of mechanical engineering in Nanjing University of Science and Technology, Jiangsu province, P.R. China, in 2006 and 2012, respectively.

He has been teaching and researching in the field of dynamic performance analysis, precision test and life/reliability analysis for function units of machine tools, especially the rolling components. Combined with rigid-flexible coupling multi-body mechanical theory, contact mechanics, etc., dynamic performance analysis and optimization design for rolling components are carried out. The research results are embedded in the related national key scientific research project. His major field of study is mechanical design, Ground mobile robot technology, measurement and control technology of mechanical system.

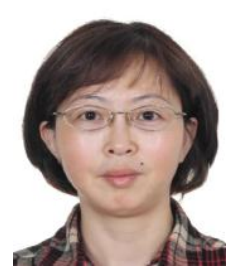

Yi Liang was born in Shaanxi, China, on August 5, 1974. Yi Liang earned her bachelor and maste degrees of mechanical engineering in Nanjing University of Science and Technology, Jiangsu province, China, in 1991 and 1998, respectively. Her major field of study is mechanical design and optimization.

She has been teaching and researching in the field of mechanical engineering since 1998 in Nanjing University of Science and Technology. The courses she teaches are mechanisms and machine theory, machine elements, and mechanical optimization for the undergraduates and postgraduates. Associate professor Yi Liang focuses on the research of mechanical design and finite element analysis of machine elements. Two of her published articles are listed here: 1). "Transmission momentary efficiency based on the D'Alembert -Lagrange equation for involutes gear," Chinese Journal of Mechanical Engineering, vol. 2, pp. 272-275, Dec. 2004; 2). "Finite element analysis of Shell of mower cutter head," Journal of Machine Design, vol. 21, pp. 253-254, Jun. 2005. Now Yi is interested in the optimization design and accuracy analysis of rolling linear guide ways. 


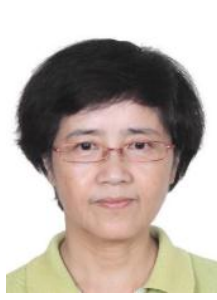

Aihua Yin was born in Shanghai, China on October 4 1953. In July 1978, Aihua Yin graduated from Donghua University, Shanghai, China, majoring in electrical engineering and automation.

She has worked in Nanjing University of Science and Technology as a teacher for more than 30 years since August 1978. Now she is an associate professor in the Department of Mechanical and Electronic Engineering. Her research interests are in the areas of mechatronics technology, robot system design, and precision measure and control technology. Her major research projects include multifunctional mobile robot, intelligent service robot and ball screw dynamic error measurement system.

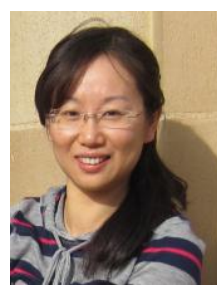

Li Zu was born in Nanjing, Jiangsu province, China on June 19, 1977. In July 1999, Li Zu received her bachelor degree in Suzhou University, Suzhou, Jiang Su Province, China in mechanical design and theory. In July 2005, $\mathrm{Li} \mathrm{Zu}$ received the doctor degree in Nanjing University of Science and Technology, Nanjing, Jiangsu Province, China in mechanical and electronic engineering.
She has worked in Nanjing University of Science and Technology as a teacher for 9 years from July 2005. Now she is an associate professor in the Dept. of Mechanical Design and Automation. From August 2012 to January 2013, She was a visiting scholar in California State University, Northridge USA. Now she is working on the National Natural Science Foundation Project - "Studies of the Load-bearing Performance of Precision Harmonic Gear within Abnormal Conditions and Design" and National Science and Technology Major Project - "Common Technology of Rolling Elements in the CNC Machine and Testing, Test Equipment Development Platform". There are two published articles here: 1). Li Zu et al., "Optimization design of the lawn mowing vehicle's blade based on aerodynamics," Advanced Materials Research, 2011, pp. 231-237. 2). Li Zu et al., "Study and design of an inchworm-1ike micro-robot walking mechanism," Lecture Notes in Electrical Engineering, vol. 123, pp. 69-78, 2011. Her research area covers mechanical design, precision drive technology and intelligent machines.

Prof. $\mathrm{Zu}$ is a member of the Association of Mechanical Engineering of Nanjing, Jiangsu Province, China. 